Laser Chem. 1988, Vol. 9, pp. 265-276

(C) 1988 Harwood Academic Publishers GmbH

Photocopying permitted by license only

Reprints available directly from the Publisher

Printed in the United Kingdom

\title{
Reactive Scattering of Oxygen Atoms with Bromine Molecules
}

\author{
N. C. FIRTH, N. W. KEANE, D. J. SMITH and R. GRICE \\ Chemistry Department, University of Manchester, Manchester M13 9PL
}

(Received 20 May, 1988)

Reactive scattering of $\mathrm{O}$ atoms with $\mathrm{Br}_{2}$ molecules has been studied at an initial translational energy $E \sim 35 \mathrm{~kJ} \mathrm{~mol}^{-1}$ using cross-correlation time-of-flight analysis with resolution improved over previous measurements. The centre-of-mass differential cross section peaks in the forward and backward directions with a higher product translational energy for backward scattering. The angular distribution traced at the peak of the product velocity distribution peaks more sharply in the forward than the backward direction but the angular distribution of product flux shows a distribution which is more nearly symmetrical about $\theta=90^{\circ}$. The observed scattering is attributed to a triplet $\mathrm{OBrBr}$ complex intermediate with a lifetime which is shorter than the period of overall rotation of the axis of the heavy $\mathrm{BrBr}$ diatomic but which is long compared with the period of vibrational and rotational motion of the light $\mathrm{O}$ atom.

KEY WORDS: Molecular beams, reactive scattering, reaction dynamics, oxygen atoms, collision complex.

\section{INTRODUCTION}

The reactions of a light atom with a heavy diatomic molecule exhibit novel dynamical features which in the case of the $F+I_{2}$ reaction, ${ }^{1}$ have been attributed to migration. This has been identified in classical trajectory calculations. ${ }^{2,3}$ Angular momentum disposal may show a strong correlation ${ }^{4}$ with the product orbital angular momentum $L^{\prime}$ and the product diatomic angular momentum $J^{\prime}$ adopting opposed directions for strongly exoergic reactions. Reactive scattering of oxygen atoms with bromine molecules exhibits a differential cross section ${ }^{5}$ at 
low initial translational energy $E \sim 1-3 \mathrm{~kJ} \mathrm{~mol}^{-1}$ which is compatible with a long-lived collision complex. At higher initial translational energy $E \sim 15-40 \mathrm{~kJ} \mathrm{~mol}^{-1}$ reactive scattering experiments ${ }^{6}$ suggest that the lifetime of the collision complex becomes comparable to the rotational period of the complex with increased scattering into the backward direction at the highest initial translational energy. However, recent kinematic analysis ${ }^{7}$ of the laboratory angular distribution of $\mathrm{OBr}$ product scattering using an RRKM model suggests that a long-lived complex mechanism may provide an adequate description up to the highest initial translational energy. In order to resolve these uncertainties concerning the detailed dynamics of the $\mathrm{O}+\mathrm{Br}_{2}$ reaction, the $\mathrm{OBr}$ reactive scattering has been remeasured using a supersonic beam of oxygen atoms seeded in He buffer gas ${ }^{8}$ with the velocity resolution improved over that employed in previous measurements. ${ }^{6}$

\section{EXPERIMENTAL}

The apparatus and experimental conditions were the same as those employed in previous experiments ${ }^{6}$ though with slightly different beam velocity distributions. The peak velocity $v_{p k}$, the full width at half maximum intensity $v_{w d}$ and the Mach number $M$ for the number

Table I Beam velocity distributions: peak velocity $v_{p k}$; full velocity width at half maximum intensity $v_{w d}$; Mach number $M$.

\begin{tabular}{llll}
\hline Beam & $v_{p k} / \mathrm{m} \mathrm{s}^{-1}$ & $v_{w d} / \mathrm{m} \mathrm{s}^{-1}$ & $M$ \\
\hline $\mathrm{O}(\mathrm{He})$ & 2300 & 670 & 5 \\
$\mathrm{Br}_{2}$ & 430 & 210 & 4 \\
\hline
\end{tabular}

density distributions are listed in Table $\mathrm{I}$. The resolution of the laboratory product velocity distributions was improved by using a time-of-flight channel width $\sim 8 \mu$ s with a chopper disc bearing five pseudorandom sequences ${ }^{9}$ and rotating at $400 \mathrm{~Hz}$.

\section{RESULTS}

The laboratory angular distribution of $\mathrm{OBr}$ reactive scattering is shown in Figure 1 and velocity distributions of $\mathrm{OBr}$ flux measured at 


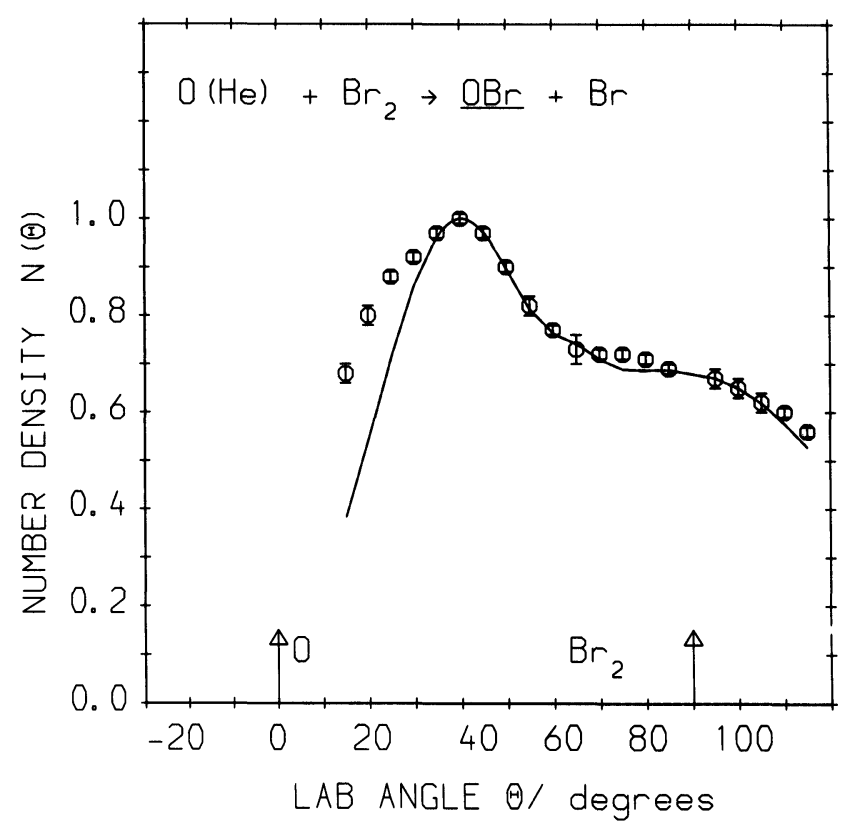

Figure 1 Laboratory angular distribution (number density) of $\mathrm{OBr}$ reactive scattering from $\mathrm{O}+\mathrm{Br}_{2}$ at an initial translational energy $E=35 \mathrm{~kJ} \mathrm{~mol}^{-1}$. Solid line shows the fit of the Entemann kinematic analysis.

each laboratory scattering angle are shown in Figure 2. Kinematic analysis of these data has been undertaken using the stochastic method of Entemann ${ }^{10}$ with a differential reaction cross section factored into a centre-of-mass angular function $T(\theta)$ and a velocity function $U(u, \theta)$ which is parametrically dependent on centre-of-mass scattering angle $\theta$

$$
I(\theta, u)=T(\theta) U(u, \theta)
$$

The form of the product velocity distribution $U(u, \theta)$ was found to depend strongly on centre-of-mass scattering angle with the highest $\mathrm{OBr}$ velocities for the backward scattering at $\theta=180^{\circ}$, lower velocity for scattering in the forward direction at $\theta=0^{\circ}$ and the lowest velocities for sideways scattering at $\theta=90^{\circ}$. The resulting angular function $T(\theta)$ and product translational energy distributions are shown in Figure 3 with the back fits of the Entemann procedure shown by a solid line in Figure 1 and broken curves in Figure 2. The form of the 

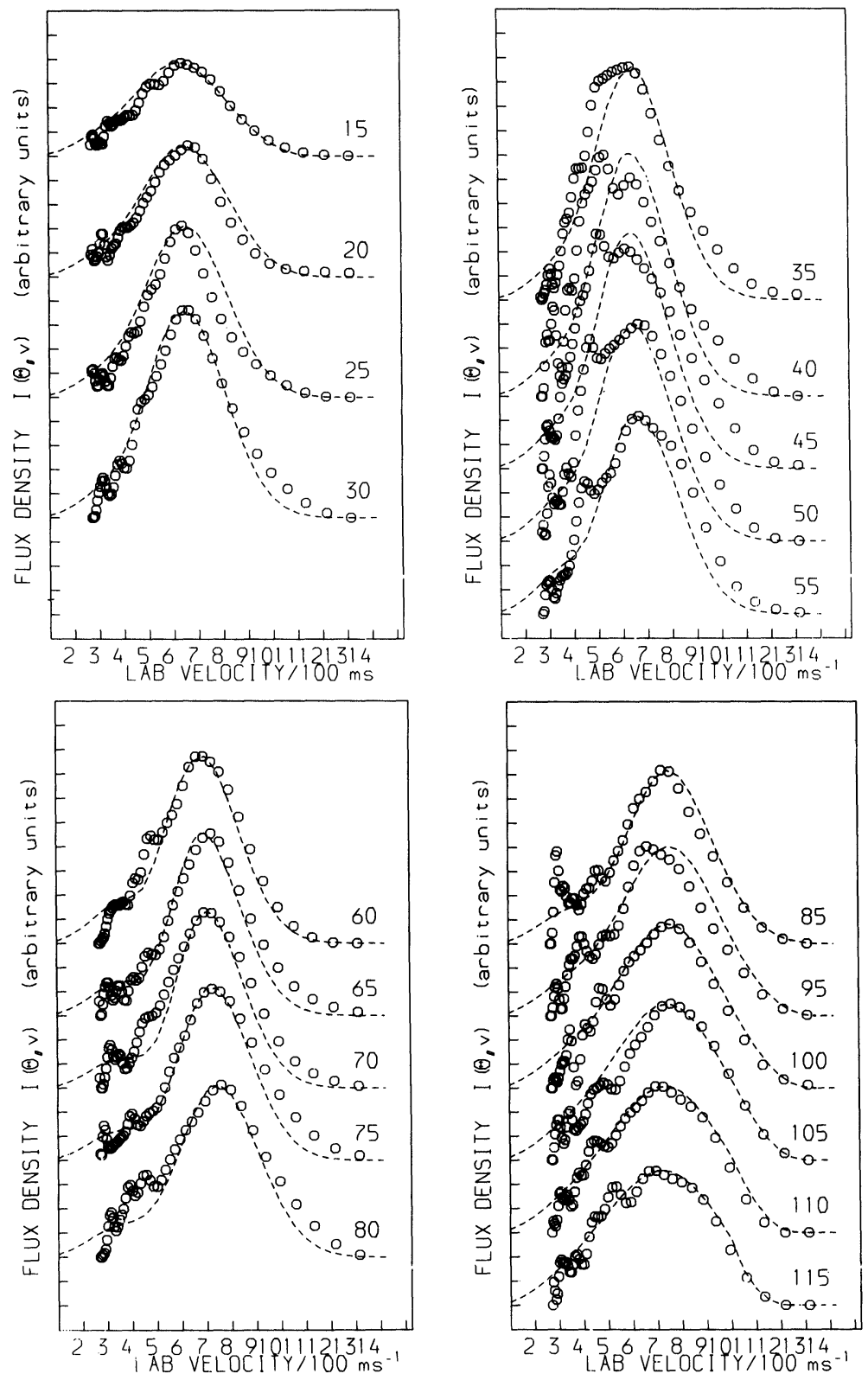

Figure 2 Laboratory velocity distributions (flux density) of reactively scattered $\mathrm{OBr}$ from $\mathrm{O}+\mathrm{Br}_{2}$ at an initial translational energy $E=35 \mathrm{~kJ} \mathrm{~mol}^{-1}$. Broken lines show the fit of the Entemann analysis. 

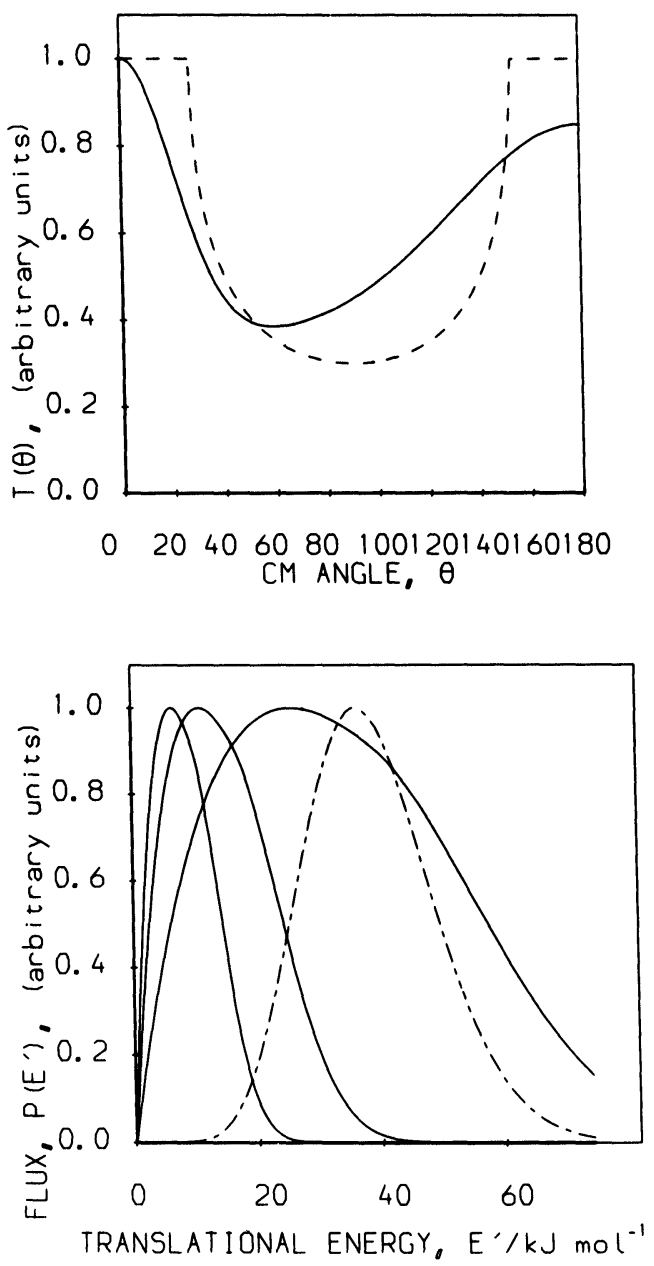

Figure 3 Angular function $T(\theta)$ and translational energy distributions $P\left(E^{\prime}\right)$ for $\mathrm{O}+\mathrm{Br}_{2}$ at an initial translational energy $E=35 \mathrm{~kJ} \mathrm{~mol}^{-1}$. Left-most product translational energy distribution corresponds to $\theta=90^{\circ}$, right-most distribution to $\theta=180^{\circ}$ and centre distribution to $\theta=0^{\circ}$. Dot-dash curve shows distribution of initial translational energy. Broken curve shows the angular distribution for a long-lived complex predicted by Eq. (7). 
angular function $T(\theta)$ is similar to that determined in previous experiments but the improved velocity resolution shows the forward scattering to be more sharply peaked with a peak height which is slightly greater than that of the backward scattering which shows a broader distribution. The peak $E_{p k}^{\prime}$ and average $E_{a v}^{\prime}$ product translational energies are listed in Table II for scattering in the forward, sideways and backward directions together with the initial translational energy $E$ and the reaction exoergicity $\Delta D_{0}$. The angular function $T(\theta)$ shown in Figure 3 is very similar in form to that determined in previous measurements ${ }^{6}$ with significant asymmetry about $\theta=90^{\circ}$. It represents the angular dependence of the $\mathrm{OBr}$ flux at the peak of the product velocity distribution. An alternative representation of the angular dependence of reactive scattering is given by the total $\mathrm{OBr}$ flux determined by integration over the product velocity distribution at each scattering angle

$$
I(\theta)=T(\theta) \int_{0}^{u_{\max }} U(u, \theta) d u
$$

The angular distribution $I(\theta)$ shown in Figure 4 differs from the angular function $T(\theta)$ shown in Figure 3 because the width and peak velocity of the product velocity distribution varies with scattering angle. The forward and backward peaks are now of equal height and the $I(\theta)$ distribution is more nearly symmetrical about $\theta=90^{\circ}$ than the $T(\theta)$ function.

\section{DISCUSSION}

The present data support previous conclusions ${ }^{6}$ that a breakdown in long-lived complex dynamics is apparent at higher initial translational energy with $\mathrm{OBr}$ product showing increased product translational energy for scattering in the backward direction. The rate constant for the $\mathrm{O}+\mathrm{Br}_{2}$ reaction at thermal energies ${ }^{11}$ is quite low $k=8.4 \times 10^{9}$ $\mathrm{dm}^{3} \mathrm{~mol}^{-1} \mathrm{~s}^{-1}$ corresponding to a total reaction cross section $Q \sim 2.5$ $\AA^{2}$. In the RRKM analysis ${ }^{12}$ this was attributed to the existence of a small activation energy $E_{a} \sim 4 \mathrm{~kJ} \mathrm{~mol}^{-1}$ with reaction being confined ${ }^{7}$ to small impact parameters $b \approx 3 \AA$. This suggests that the formation of the reaction intermediate in the entrance valley of the triplet potential energy surface may be modelled by an abrupt transition as shown in 

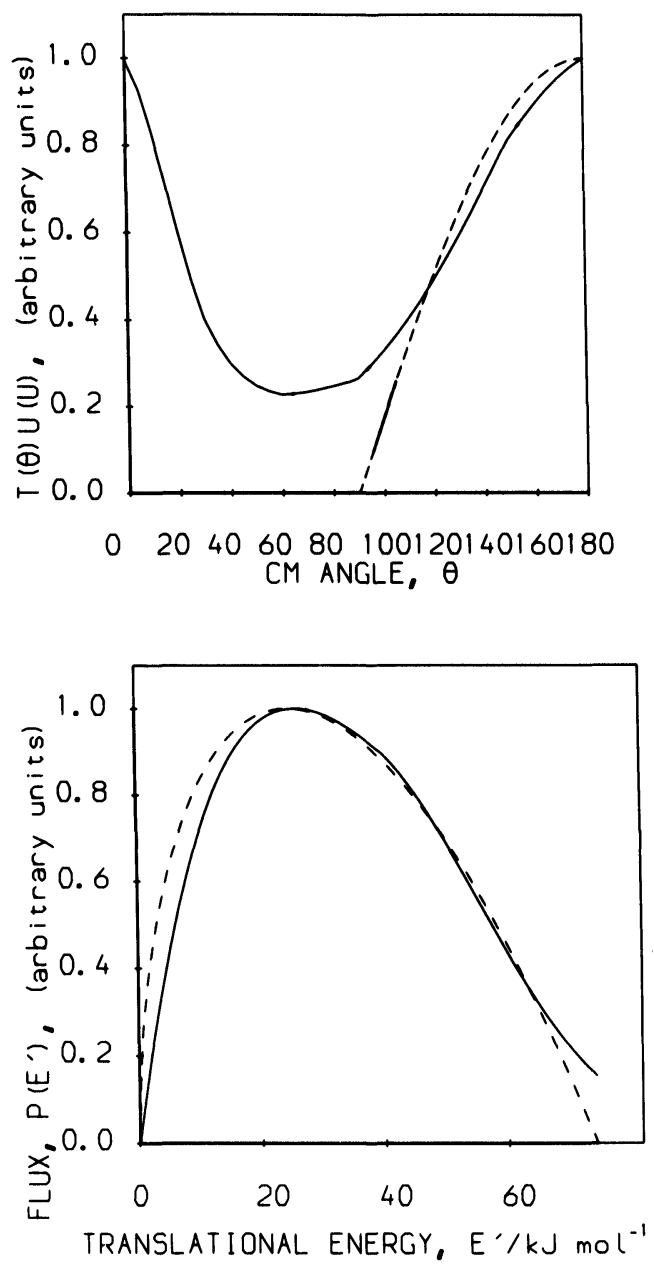

Figure 4 Angular distribution $I(\theta)$ and translational energy distribution $P\left(E^{\prime}\right)$ for backward scattering at $\theta=180^{\circ}$. Broken angular curve shows cosine distribution of Eq. (12) and broken energy curve shows microcannonical RRHO distribution of Eq. (9).

Figure 5 for a bent $\mathrm{O}-\mathrm{Br}-\mathrm{Br}$ complex. This shows a collision between an atom $A$ with impact parameter $b$ and a diatomic molecule $B C$, when the $A$ atom reaches the $A B$ bond length of the bent $A B C$ transition state with interbond angle $\beta$. The initial orbital angular momentum $L$ lies perpendicular to the plane of collision and the orientation of the $B C$ diatomic is defined by spherical polar angles $\alpha$ and $\phi$ with respect to 


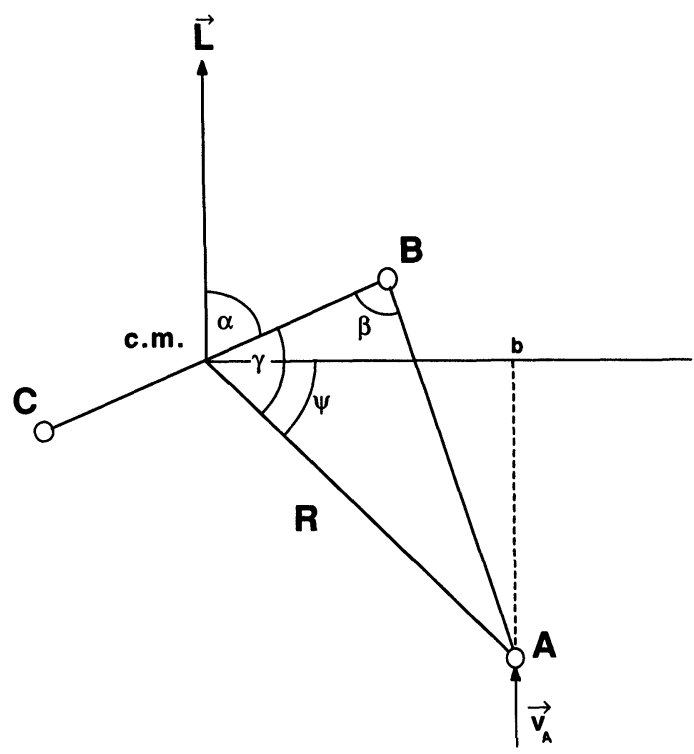

Figure 5 Initial configuration of a bent $A B C$ complex with interbond angle $\beta$ and impact parameter $b$. The initial orbital angular momentum $L$ is perpendicular to the plane of collision.

L. The complex is formed with the $A$ atom lying in the plane of collision at a fixed radial distance $R$ from the centre of mass of the $B C$ molecule, where

$$
R=\left[r_{A B}^{2}+\left(m_{C} r_{B C} / m_{B C}\right)^{2}-2 r_{A B}\left(m_{C} r_{B C} / m_{B C}\right) \cos \beta\right]^{1 / 2}
$$

If the radius $R$ makes angle $\psi$ with the impact parameter $b$ at the moment of impact then $b=R \cos \psi$. The initial orientation of the $A B C$ complex is then specified by the spherical polar angles $\alpha, \phi$ defining the orientation of the $B C$ axis subject to the limits $|\cos \alpha| \leqslant \sin \gamma$ and $b \leqslant R$ where

$$
\tan \gamma=\sin \beta /\left[m_{C} r_{B C} /\left(m_{B C} r_{A B}\right)-\cos \beta\right]
$$

The probability of any initial orientation of the $B C$ axis per unit solid angle is uniform

$$
P(\cos \alpha, \phi)=1 /(4 \pi)
$$

For specified values of the impact parameter $b$ and the polar angle $\alpha$ there are two possible values of the azimuthal angle $\phi$ except in the 
limit $|\cos \alpha|=\sin \gamma$ when the plane of the $A B C$ complex lies initially perpendicular to the plane of collision.

If we now consider the case of a light atom $A$ and heavy diatomic molecule $B C$, the subsequent motion of the complex ${ }^{13}$ may be approximated as that of a symmetric top molecule with the top axis identified with the $B C$ diatomic axis. The angular distribution generated by the precession of a long-lived symmetric top complex ${ }^{14}$ is given by

$$
\left.T(\theta, \alpha)=1 / \pi\left(\sin ^{2} \theta-\cos ^{2} \alpha\right)^{1 / 2}\right] \text { for } \cos ^{2} \alpha \leqslant \sin ^{2} \theta
$$

and zero otherwise. The probability of $\cos \alpha$ is uniform according to Eq. (5) for $|\cos \alpha| \leqslant \sin \gamma$ and the resulting angular distribution ${ }^{13}$ is then given by

$$
\begin{aligned}
& T(\theta)=(2 / \pi) \operatorname{arc} \sin (\sin \gamma / \sin \theta) \text { for } \sin \theta>\sin \gamma \\
& T(\theta)=1 \text { for } \sin \theta \leqslant \sin \gamma
\end{aligned}
$$

This form of angular distribution applies independently of the impact parameter $b \leqslant R$ under the present model for a long-lived collision complex since precession through many rotational periods generates an angular distribution which is independent of the initial value of the azimuthal angle $\phi$. When the lifetime of the complex becomes comparable to its rotational period, however, the angular distribution will depend on the initial value of $\phi$ and hence upon the impact parameter $b$.

If the $\mathrm{OBrBr}$ triplet complex is assumed to have bond lengths $r_{\mathrm{OBr}}=$ $1.8 \AA, r_{\mathrm{BrBr}}=2.4 \AA$ and an interbond angle $\beta=135^{\circ}$ in accord with the value previously estimateed ${ }^{15}$ for $\mathrm{F}+\mathrm{Br}_{2}$, then Eqs. (3) and (4) yield $R$ $=2.8 \AA$ and $\gamma=27^{\circ}$. The angular distribution predicted by Eq. (7) and shown by a broken curve in Figure 3, exhibits quite sharp forward and backward peaking which is in fair agreement with the forward peak of the angular function $T(\theta)$ but which is narrower than the backward peak. Rather better agreement is found with the angular distribution $I(\theta)$ in Figure 4 which has a narrower backward peak than $T(\theta)$ in Figure 3. The narrow peak exhibited for forward scattering at $\theta=0^{\circ}$ suggests that this arises from precession of $\mathrm{OBrBr}$ complexes formed in collisions at large impact parameters. Indeed the peak product translational energy for forward scattering listed in Table II is in good agreement with the value predicted for the maximum centrifugal barrier $^{16}$

$$
B_{m}^{\prime}=\left(\mu / \mu^{\prime}\right) E
$$


Table II Reaction energetics: reactant translational energy $E$; peak product translational energy $E_{p k}^{\prime}$; average product translational energy $E_{a v}^{\prime}$; reaction exoergicity $\Delta D_{0}$.

\begin{tabular}{|c|c|c|c|c|c|c|c|}
\hline \multirow{2}{*}{$\begin{array}{l}E / \mathrm{kJ} \\
\mathrm{mol}^{-1}\end{array}$} & \multicolumn{3}{|c|}{$E_{p k}^{\prime} / \mathrm{kJ} \mathrm{mol}^{-1}$} & \multicolumn{3}{|c|}{$E_{a v}^{\prime} / \mathrm{kJ} \mathrm{mol}^{-1}$} & \multirow{2}{*}{$\begin{array}{l}\Delta D_{0} / \mathrm{kJ} \\
\mathrm{mol}^{-1}\end{array}$} \\
\hline & $\theta=0^{\circ}$ & $\theta=90^{\circ}$ & $\theta=180^{\circ}$ & $\theta=0^{\circ}$ & $\theta=90^{\circ}$ & $\theta=180^{\circ}$ & \\
\hline 35 & 11 & 8 & 25 & 16 & 11 & 39 & 40 \\
\hline
\end{tabular}

where $\mu, \mu^{\prime}$ denote the reactant and product reduced masses, which yields $B_{m}^{\prime} \sim 13 \mathrm{~kJ} \mathrm{~mol}^{-1}$. In contrast the higher product translational energy observed for scattering in the backward direction $\theta=180^{\circ}$ suggests that this arises primarily from collisions at small impact parameters with low initial orbital angular momentum. In this case the product translational energy distribution might be expected to be in accord with the microcannonical RRHO prior distribution ${ }^{17}$ which takes no account of angular momentum conservation

$$
P\left(f^{\prime}\right)=\left(3^{3 / 2} / 2\right) f^{\prime 1 / 2}\left(1-f^{\prime}\right)
$$

where $f^{\prime}=E^{\prime} /\left(E+\Delta D_{0}\right)$. That this is indeed the case is demonstrated in Figure 4 where the RRHO prior distribution shown by a broken curve is in excellent agreement with the product translational energy distribution for backward scattering shown by a solid curve. It might also be anticipated that the angular distribution for backward scattering arising from small impact parameter collisions should reflect the reactant scattering angle of the light incident $\mathrm{O}$ atom with little contribution from rotation of the heavy $\mathrm{Br}_{2}$ molecule. It is apparent from Figure 5 that this gives

$$
b=R \sin \theta
$$

Following the formula ${ }^{17}$

$$
I(\theta)=-(b / \sin \theta) d b / d \theta
$$

for the differential reaction cross section yields

$$
I(\theta)=-R^{2} \cos \theta \quad \pi / 2 \leqslant \theta \leqslant \pi
$$

and zero otherwise. The cosine angular distribution predicted by the hard hemisphere model of Eq. (12), shown by a broken curve in Figure 4 is again in excellent agreement with the experimental angular distribution $I(\theta)$ in the backward hemisphere shown by a solid curve, though there is rather less close agreement with the angular function $T(\theta)$ shown in Figure 3. 
The present results are consistent with reactive scattering via a short-lived $\mathrm{OBrBr}$ collision complex on the triplet potential energy surface. ${ }^{18}$ The direction of scattering is related to impact parameter with backward scattering arising from small impact parameter collisions and forward scattering from larger impact parameters. The product translational energy distribution varies with scattering angle. Precession of complexes ${ }^{13}$ with significant initial orbital angular momentum $L \sim 160 \hbar$ gives lower translational energy for scattering in the forward direction than the more direct dissociation of complexes with low initial orbital angular momentum scattering in the backward direction. Thus the collisional lifetime for most complexes is less than the period of rotation of the principal axis of the $\mathrm{OBrBr}$ complex which is essentially the axis of the heavy $\mathrm{BrBr}$ diatomic. However, the product translational energy distributions show clear evidence of energy randomisation prior to complex dissociation which may be associated with the much shorter period of the light $O$ atom vibrational and rotational motion. The approximate symmetry about $\theta=90^{\circ}$ of the angular flux distribution of reactive scattering therefore arises in part from the near equality of scattering from small and larger impact parameter collisions. The $\mathrm{OBrBr}$ complexes are long-lived with respect to the rapid $\mathrm{O}$ atom motion but not with respect to $\mathrm{Br}-\mathrm{Br}$ rotation. This dichotomy is prevalent in the reaction dynamics of a light atom with a heavy diatomic ${ }^{2-4}$ and explains why the overall scattering shows many characteristics of long-lived collision dynamics. $^{7}$

\section{Acknowledgement}

Support of this work by SERC is gratefully acknowledged.

\section{References}

1. N. C. Firth, N. W. Keane, D. J. Smith and R. Grice, Faraday Discuss. 84, 53 (1988).

2. I. W. Fletcher and J. C. Whitehead, Faraday Trans. 2 78; 1165 (1982); 80, 985 (1984).

3. M. I. Urrecha, F. Castano and J. Iturbe, Faraday Trans. 2 82, 1077 (1986).

4. S. K. Kim and D. R. Herschbach, Faraday Discuss. 84, 159 (1988).

5. D. D. Parrish and D. R. Herschbach, J. Amer. Chem. Soc. 95, 6133 (1973); D. St.

A. G. Radlein, J. C. Whitehead and R. Grice, Molec. Phys. 29, 1813 (1975).

6. D. P. Fernie, D. J. Smith, A. Durkin and R. Grice, Molec. Phys. 46, 41 (1982).

7. P. A. Elofson, K. Rynefors and L. Holmlid, Chem. Phys. 118, 1 (1987).

8. P. A. Gorry and R. Grice, J. Phys. E. 12, 857 (1979). 
9. C. V. Nowikow and R. Grice, J. Phys. E. 12, 515 (1979).

10. E. A. Entemann and D. R. Herschbach, Discuss. Faraday Soc. 44, 289 (1967).

11. M. A. A. Clyne, P. B. Monkhouse and L. W. Townsend, Intl. J. Chem. Kinet. 8, 425 (1976).

12. K. Rynefors, L. Holmlid and P. A. Elofson, Chem. Phys. 118, 417 (1987).

13. N. W. Keane and R. Grice, Molec. Phys. 61, 869 (1987).

14. I. Halpern and V. M. Strutinski, Proc. 2nd Int. Conf., Peaceful Uses of Atomic Energy, Geneva, p. 408 (1958).

15. N. C. Firth and R. Grice, Molec. Phys. 60, 1273 (1987).

16. S. A. Safron, N. D. Weinstein, D. R. Herschbach and J. C. Tully, Chem. Phys. Lett. 12, 564 (1972).

17. R. D. Levine and R. B. Bernstein, Molecular Reaction Dynamics and Chemical Reactivity (Oxford University Press, 1987).

18. R. Grice, Chem. Soc. Rev. 11, 1 (1982). 